\title{
Novel oral plasminogen activator inhibitor-1 inhibitor TM5275 attenuates hepatic fibrosis under metabolic syndrome via suppression of activated hepatic stellate cells in rats
}

\author{
RYUICHI NOGUCHI $^{1}$, KOSUKE KAJI ${ }^{1}$, TADASHI NAMISAKI ${ }^{1}$, KEI MORIYA ${ }^{1}$, HIDETO KAWARATANI ${ }^{1}$, \\ MITSUTERU KITADE ${ }^{1}$, HIROAKI TAKAYA ${ }^{1}$, YOSUKE AIHARA ${ }^{1}$, AKITOSHI DOUHARA ${ }^{1}$, \\ KIYOSHI ASADA ${ }^{1}$, NORIHISA NISHIMURA ${ }^{1}$, TOSHIO MIYATA ${ }^{2}$ and HITOSHI YOSHIJI ${ }^{1}$ \\ ${ }^{1}$ Department of Gastroenterology, Nara Medical University, Kashihara, Nara 634-8522; \\ ${ }^{2}$ United Centers for Advanced Research and Translational Medicine, Tohoku University \\ Graduate School of Medicine, Sendai, Miyagi 980-8575, Japan
}

Received March 28, 2020; Accepted July 3, 2020

DOI: $10.3892 / \mathrm{mmr} .2020 .11360$

\begin{abstract}
An orally bioavailable small molecule inhibitor of plasminogen activator inhibitor-1 (PAI-1) is currently being clinically assessed as a novel antithrombotic agent. Although PAI-1 is known to serve a key role in the pathogenesis of metabolic syndrome (MetS) including nonalcoholic steatohepatitis (NASH), the pharmacological action of an oral PAI-1 inhibitor against the development of MetS-related liver fibrosis remains unclear. The current study was designed to explicate the effect of TM5275, an oral PAI-1 inhibitor, on MetS-related hepatic fibrogenesis. The in vivo antifibrotic effect of orally administered TM5275 was investigated in two different rat MetS models. Fischer 344 rats received a choline-deficient L-amino-acid-defined diet for 12 weeks to induce steatohepatitis with development of severe hepatic fibrosis. Otsuka Long-Evans Tokushima Fatty rats, used to model congenital diabetes, underwent intraperitoneal injection of porcine serum for 6 weeks to induce hepatic fibrosis under diabetic conditions. In each experimental model, TM5275 markedly ameliorated the development of hepatic fibrosis and suppressed the proliferation of activated hepatic stellate cells (HSCs). Additionally, the hepatic production of tumor growth factor (TGF)- $\beta 1$ and total collagen was suppressed. In vitro assays revealed that TGF- $\beta 1$ stimulated the upregulation of Serpine1 mRNA expression, which was inhibited by TM5275 treatment in cultured HSC-T6 cells, a rat HSC cell line. Furthermore, TM5275 substantially attenuated the TGF- $\beta 1$-stimulated
\end{abstract}

Correspondence to: Dr Kosuke Kaji, Department of Gastroenterology, Nara Medical University, 840 Shijo-cho, Kashihara, Nara 634-8522, Japan

E-mail:kajik@naramed-u.ac.jp

Key words: plasminogen activator inhibitor-1, nonalcoholic steatohepatitis, liver fibrosis, hepatic stellate cell, transforming growth factor- $\beta$ proliferative and fibrogenic activity of HSCs by inhibiting AKT phosphorylation. Collectively, TM5275 demonstrated an antifibrotic effect on liver fibrosis in different rat MetS models, suppressing TGF- $\beta 1$-induced HSC proliferation and collagen synthesis. Thus, PAI-1 inhibitors may serve as effective future therapeutic agents against NASH-based hepatic fibrosis.

\section{Introduction}

Nonalcoholic steatohepatitis (NASH) is the inflammatory subtype nonalcoholic fatty liver disease (NAFLD). It is a representative phenotype of metabolic syndrome (MetS) in the liver and is involved in disease progression leading to cirrhosis and need for liver transplantation (1-4). Emerging evidences claim that the degree of hepatic fibrosis is considered the most crucial predictor of overall mortality in patients with NASH $(5,6)$. This indicates that the ultimate medical goal in the treatment of NASH would be to clinically prevent the development of liver fibrosis. Thus, a variety of pharmacological strategies have been proposed, but no evidence-based pharmacotherapies have been established for MetS-related liver fibrosis including NASH.

Plasminogen activator inhibitor-1 (PAI-1), a member of the serine protease inhibitor (serpin) gene family, is the major physiologic regulator of the plasmin-based pericellular proteolytic cascade $(7,8)$. PAI-1 is synthesized by numerous types of cells including cardiac myocytes, lipocytes, vascular endothelial cells, macrophages, and fibroblasts. It also acts as a potent inhibitor of fibrinolysis by regulating urokinase plasminogen activator (uPA)/tissue plasminogen activator (tPA)/plasmin/matrix metalloproteinase (MMP) proteolytic activity, and fibrin levels. It is therefore extensively involved in fibrogenesis in multiple organs such as skin, lung, heart, kidney, and liver (9-16). Indeed, fibrotic livers show a significantly higher level of PAI-1 (13), while PAI-1 deficiency ameliorates bile duct ligation-induced cholestatic liver fibrosis with promotion of collagen degradation (14). A recent clinical report has demonstrated that higher plasma levels of PAI-1 were strongly associated with NASH severity 
in obese patients (15). Additionally, siRNA-mediated inhibition of PAI-1 was shown to exert a protective effect on murine liver fibrosis and to alleviate the fibrotic properties in hepatic stellate cells (HSCs), a major component of the fibrotic process in the liver (16). This evidence, therefore, further supports the concept that PAI-1 is possible to be suitable as a therapeutic target for the liver fibrosis.

TM5275 55-chloro-2-[(\{2-[4-(diphenylmethyl)piperazin-1-yl] -2-oxoethoxy\}acetyl)amino]benzoate\} is an orally bioavailable small molecule PAI-1 inhibitor $(17,18)$. It exerts powerful antithrombotic activity in both rodents and nonhuman primates (cynomolgus monkey) (17,18). Of great importance, TM5275 does not interfere with other serpin/serine protease systems including 1-antitrypsin/trypsin and $\alpha 2$-antiplasmin/plasmin and causes no obvious toxicity in the liver, kidney, hematopoietic system, central nervous system, or cardiovascular system of rodents and primates (17-19). Hence, it has no detrimental effects on hemostatic function, which are the most common adverse effects of anticoagulation agents; this suggests that TM5275 specifically acts on PAI-1 with limited toxicity (17-19). Actually, TM5441, a derivative of TM5275, has been reported to show a suppressive effect in murine fatty liver models $(20,21)$. However, the direct effect of TM5275 on liverfibrosis development under the condition of MetS in conjunction with the proliferation and activation of HSCs has not been investigated.

The present study aimed to explore the effect of the PAI-1 inhibitor TM5275 in improving the pathology of MetS-based fibrosis and to determine the therapeutic mechanisms in HSC biology using two rat liver fibrosis models under the condition of MetS: Choline-deficient, L-amino acid-defined diet (CDAA)-fed rats or porcine serum (PS)-induced fibrotic Otsuka Long-Evans Tokushima Fatty (OLETF) diabetic rats.

\section{Materials and methods}

Animals and reagents. Six-week-old male Fischer-344 rats, ten-week-old male OLETF and Long-Evans Tokushima Otsuka (LETO), littermate controls for OLETF, were purchased from Japan SLC, Inc. The PAI-1 inhibitor TM5275 was provided by Toshio Miyata (Tohoku University Graduate School of Medicine). Recombinant rat PAI-1 was purchased from PeproTech. Conventional chemical agents were obtained from Nacalai Tesque. The rat HSC cell line HSC-T6 was purchased from the Japanese Cancer Research Resources Bank.

Animal treatment. Two different in vivo models of liver fibrosis were established in rats: CDAA-fed rats or PS-mediated fibrotic OLETF rats. In the CDAA-fed model, the Fisher-344 rats received a CDAA diet (CLEA Japan) for 12 weeks, and the treatment group $(\mathrm{n}=6)$ was orally administered $50 \mathrm{mg} / \mathrm{kg} / \mathrm{day}$ of TM5275 in the drinking water. A positive control group $(n=6)$ was administered the same amount of vehicle during the CDAA feeding period, and the negative control group $(n=6)$ consisted of the rats fed a choline-supplemented amino acid (CSAA) diet (CLEA Japan) and treated with vehicle. In the PS-mediated fibrotic OLETF rats, the OLETF rats were intraperitoneally administered $1 \mathrm{ml} / \mathrm{kg}$ PS (Rockland Immunochemicals, Inc.) twice a week for 6 weeks. Treatment groups $(n=6$ each) received administration of 50 or
$100 \mathrm{mg} / \mathrm{kg} / \mathrm{day}$ of TM5275 in the drinking water. A positive control group $(n=6)$ was administered the same amount of vehicle during the PS injection. As a negative control group $(n=6)$, LETO rats received a PS injection and were treated with vehicle in the same manner as the positive control group. All rats were housed in stainless steel mesh cages under the following controlled conditions: Temperature: $23 \pm 3^{\circ} \mathrm{C}$; relative humidity: $50 \pm 20 \%$; $10-15$ air changes/h; and $12-\mathrm{h}$ day/night cycle. The rats had ad libitum access to tap water throughout the study period. At the end of the experiment, all rats underwent the following procedures: Euthanasia by intraperitoneal injection of pentobarbital sodium $(200 \mathrm{mg} / \mathrm{kg})$, opening of the abdominal cavity, blood collection via puncture of the aorta and harvesting of liver for histological and molecular evaluation. Rats were subsequently decapitated to assure for death.

Serum markers, such as alanine aminotransferase (ALT), were assessed by routine laboratory methods. All animal procedures were performed according to the criteria outlined in the Guide for the Care and Use of Laboratory Animals prepared by the National Academy of Sciences. All experiments were approved by the Animal Care and Use Committee of Nara Medical University (Protocol no. 10034).

Estimation of glycemic status. At the experiment's conclusion, insulin sensitivity and insulin resistance were evaluated according to the quantitative insulin sensitivity check index (QUICKI) and the homeostasis model assessment of insulin resistance (HOMA-IR), respectively, as previously described (22).

Immunohistochemical staining and semi-quantification. Liver specimens were fixed in $10 \%$ formalin and embedded in paraffin. Sections of $5-\mu \mathrm{m}$ thickness were stained with hematoxylin and eosin (H\&E) and Sirius-Red. Histological scores for steatosis, lobular inflammation and hepatocyte ballooning were calculated according to the NAFLD Activity Score (23); steatosis $(0 ;<5 \%, 1 ; 5-33 \%, 2 ;>33-66 \%, 3 ;>66 \%)$, lobular inflammation ( 0 ; no foci, 1 ; <2 foci/200-fold, 2; 2-4 foci/200-fold, 3 ; $>4$ foci/200-fold), hepatocyte ballooning ( 0 ; none, 1 ; few balloon cells, 2 ; many cells/prominent ballooning). These scoring were performed for 5 fields per each section. A primary antibody against $\alpha$-SMA (ab124964; 1:1,000 dilution) (Abcam) was applied for immunostaining. Specifically, staining was performed according to the supplier's recommendations. The quantitative analyses for liver fibrosis and $\alpha$-SMA-positive area were performed for 5 fields per each section in high-power fields at 400 -fold magnification by applying NIH ImageJ software.

Measurement of protein levels of hepatic collagen content and $T G F-\beta 1$. After equalizing the protein concentration from frozen liver samples to $200 \mathrm{mg}$, hepatic collagen content and TGF- $\beta 1$ protein were measured using Sircol Soluble Collagen Assays (Biocolor Ltd.) and TGF- $\beta 1$ ELISA kits (Bender MedSystems $\mathrm{GmbH}$ ) according to the manufacturer's instructions.

Cell culture and WST-1 assay. Rat HSCs (HSC-T6) (cat no. SCC069) were purchased from Merck KGaA. Cells were cultured in Dulbecco's Modified Eagle's Medium (Nacalai) supplemented with $10 \%$ fetal bovine serum and $2 \mathrm{mM}$ 
1-glutamine in a $95 \%$ air $/ 5 \% \mathrm{CO}_{2}$ humidified atmosphere at $37^{\circ} \mathrm{C}$. Mycoplasma testing was performed using MycoProbe ${ }^{\circledR}$ Mycoplasma Detection kit (R\&D Systems, Inc.) according to the manufacturer's protocol. For the assay, HSC-T6 cells $\left(1 \times 10^{4}\right)$ were seeded in a 96-well plate. Subsequently, the cells were treated with different concentrations of recombinant rat PAI-1 or $10 \mathrm{ng} / \mathrm{ml}$ of rat TGF- $\beta 1$ (Abcam) and/or different doses of TM5275 for $24 \mathrm{~h}$. Premix WST-1 Cell Proliferation Assay System (Takara Bio Inc.) was applied for cell proliferation analysis.

RNA extraction and real-time polymerase chain reaction. Total RNA was isolated from the liver tissues and HSC-T6 cells using a RNeasy Mini kit (Qiagen). HSC-T6 cells were incubated in the presence or absence of rat TGF- $\beta 1(10 \mathrm{ng} / \mathrm{ml})$ and/or TM5275 $(100 \mu \mathrm{M})$ for $12 \mathrm{~h}$. The mRNA levels of Tgfbl, Collal, and Serpinel were measured by quantitative polymerase chain reaction (qPCR) using an Applied Biosystems StepOnePlus ${ }^{\mathrm{TM}}$ Real-Time PCR ${ }^{\circledR}$ system (Applied Biosystems). Primer sequences were as follows: $T g f b 1$, forward 5'-CGG CAG CTG TAC ATT GAC TT-3' and reverse 5'-AGC GCA CGA TCA TGT TGG AC-3'; Collal, forward 5'-AGC TCC TGG GCC TAT CTG ATG A-3' and reverse 5'-AAT GGT GCT CTG AAA CCC TGA TG-3'; and Serpine1, forward 5'-AGG GGC AGC AGA TAG ACA GA-3' and reverse 5'-CAC AGG GAG ACC CAG GTA AA-3'. Relative gene expression levels were determined using glyceraldehyde-3-phosphate dehydrogenase (Gapdh): Forward 5'-CGA CCA CTT TGT CAA GCT CA-3' and reverse 5'-AGG GGA GAT TCA GTG TGG TG-3'; as the internal control. The predicted cycle threshold (CT) values were exported for analysis. All reactions were performed using a 1:10 diluted cDNA while mRNA expression levels were estimated using the $2^{\Delta \Delta C T}$ method by normalization to Gapdh and showed relative values to each control group.

Protein extraction and western blotting. Proteins were extracted from $10^{6}$ cultured HSC-T6 cells. HSC-T6 cells were incubated in the presence or absence of rat TGF- $\beta 1(10 \mathrm{ng} / \mathrm{ml})$ and/or TM5275 $(100 \mu \mathrm{M})$ for $12 \mathrm{~h}$. For this purpose, T-PER Tissue Protein Extraction Reagent as lysis buffer supplemented with proteinase and phosphatase inhibitors (Thermo Fisher Scientific Inc.) was used. The protein concentration was measured by protein assay (BioRad), and all samples were normalized to $50 \mu \mathrm{g}$. Cellular proteins were separated by SDS-PAGE (Thermo Fisher Scientific Inc.) and transferred to an Invitrolon PVDF membrane (Thermo Fisher Scientific Inc.). And then membranes were blocked with 5\% bovine serum albumin in Tris-buffered saline with Tween-20 for $1 \mathrm{~h}$. The AKT (\#9272; 1:1,000 dilution), phosphorylated AKT (Ser473) (p-AKT) (\#9271; 1:1,000 dilution), extracellular signal-regulated kinase (ERK1/2) (\#9102; 1:1,000 dilution), phosphorylated ERK1/2 (Thr202/Tyr204) (p-ERK1/2) (\#4370; 1:1,000 dilution), or SMAD2/3 (\#3102; 1:1,000 dilution), phosphorylated SMAD2 (Ser465/Ser467)/3 (Ser423/425) (p-SMAD2/3) (\#8828; 1:1,000 dilution), GAPDH (\#5174; 1:1,000 dilution) and $\beta$-actin (Actin) (\#4967; 1:10,000 dilution) (Cell Signaling Technology) were used as primary antibodies Amersham ECL IgG, HRP-linked $\mathrm{F}(\mathrm{ab}) 2$ fragment (GE Healthcare Life Sciences; 1:5,000 dilution) was applied as a secondary antibody. The bands were visualized using Clarity Western ECL Substrate (BioRad).
Statistical analysis. The data were subjected to a one-way analysis of variance followed by Bonferroni's multiple-comparison test, where appropriate. Bartlett's test was used to determine the homology of variance. All tests were two-tailed, and $\mathrm{P}<0.05$ was considered to indicate a statistically significant difference.

\section{Results}

TM5275, a PAI-1 inhibitor, attenuates the progression of liver fibrosis in CDAA-fed rats. Initially, we assessed the effect of a PAI-1 inhibitor in the in vivo development of liver fibrosis in CDAA-fed rats (Fig. 1A). As shown in Fig. 1B, 12 weeks of CDAA feeding induced prominent hepatic steatosis and fibrosis with collagen fiber deposition, which were histologically indicated by $\mathrm{H} \& \mathrm{E}$ and Sirius-Red staining, respectively, as principal phenotypic changes of NASH. Moreover, immunohistochemistry for $\alpha$-SMA revealed that CDAA-fed mice showed an increase in immunopositive activated HSCs compared with CSAA-fed negative control rats. Continuous oral administration of TM5275, a pharmacological PAI-1 inhibitor, did not affect the histological changes in CDAA-fed rats according to the NAFLD activity score, which includes steatosis, lobular inflammation, and hepatocyte ballooning (Fig. 1B and C). By contrast, treatment with TM5275 significantly attenuated liver fibrosis development in CDAA-fed rats (Fig. 1B). Computer-assisted semiquantitative analysis demonstrated a decrease to one-half the fibrotic area of the liver in CDAA-fed rats treated with TM5275 compared with vehicle-treated rats (Fig. 1D). The CDAA-fed rats who were administered TM5275 also showed a marked decrease in $\alpha$-SMA-immunopositive areas compared with those treated with vehicle in accordance with attenuated liver fibrosis (Fig. 1E). This suppressed fibrogenesis coincided with decreases in the hepatic expression of profibrogenic genes, including Tgfbl and Collal (Fig. 2A and B), and reduced hepatic levels of TGF- $\beta 1$ and total collagen (Fig. $2 \mathrm{C}$ and D). These findings suggest that the PAI-1 inhibitor TM5275 can potentially inhibit liver fibrosis development without affecting hepatic steatosis and inflammation, especially in CDAA-induced steatohepatitis.

TM5275 exerts an inhibitory effect on porcine serum-induced liver fibrosis in diabetic OLETF rats. To evaluate whether the PAI-1 inhibitor TM5275 exerts antifibrotic effects independently of its antisteatotic and anti-inflammatory properties, we next examined the effect of TM5275 on porcine serum (PS)-induced liver fibrosis development in OLETF genetically engineered diabetic rats (Fig. 3A). We have reported that this model could induce progression of liver fibrosis with limited hepatic steatosis and inflammation (24). As shown in Fig. 3B, OLETF rats showed impaired insulin sensitivity and insulin resistance, which were calculated according to QUICKI and HOMA-IR, respectively, and these impairments remained unchanged by 12 weeks of treatment with TM5275. Moreover, OLETF rats showed a mild elevation in serum alanine aminotransferase (ALT) as a result of hepatic steatosis, and neither low-nor high-dose treatments with TM5275 significantly altered the increased serum ALT level in OLETF rats. As previously reported, PS administration 
A

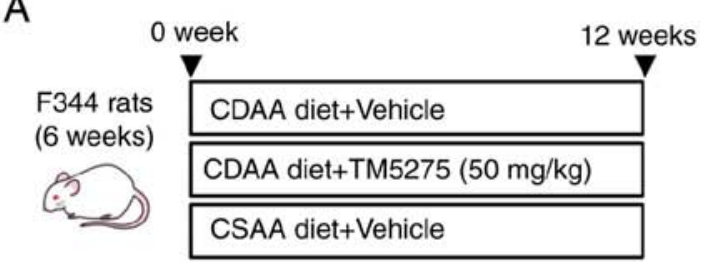

C

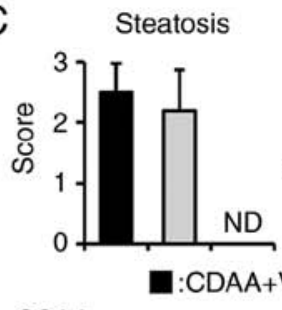

Inflammation
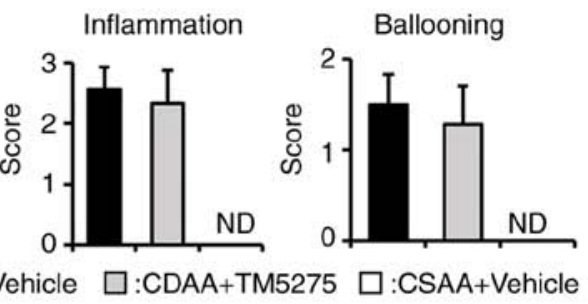

B

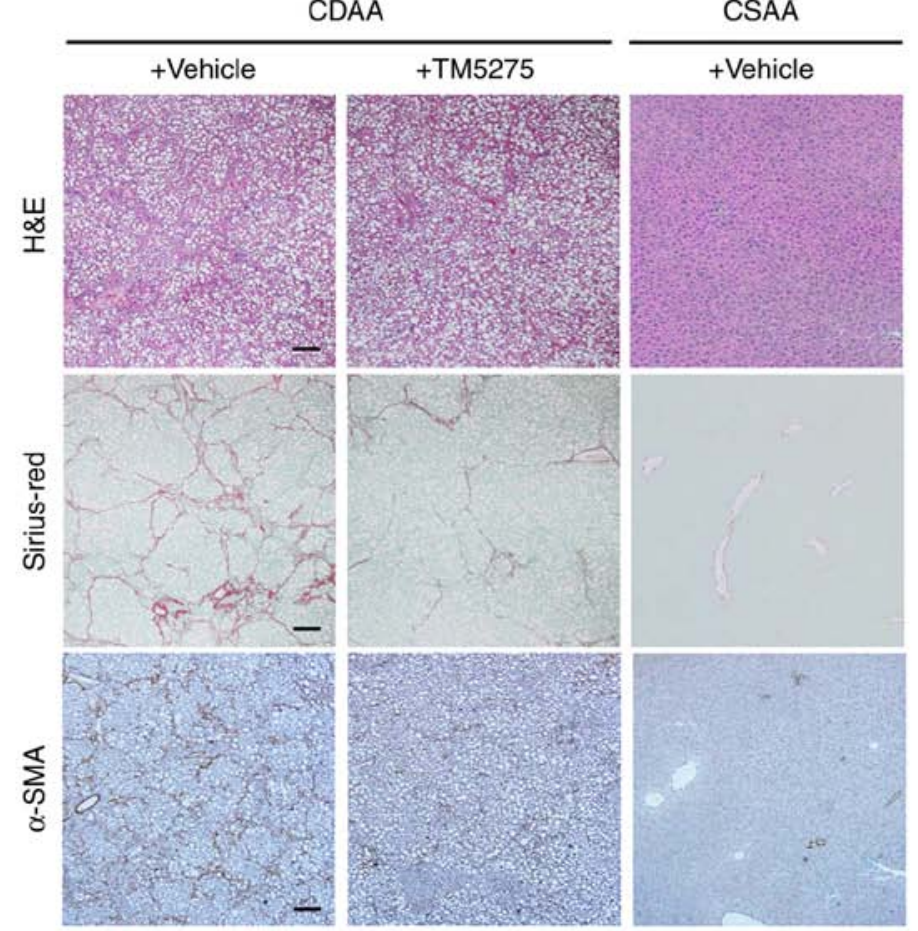

D

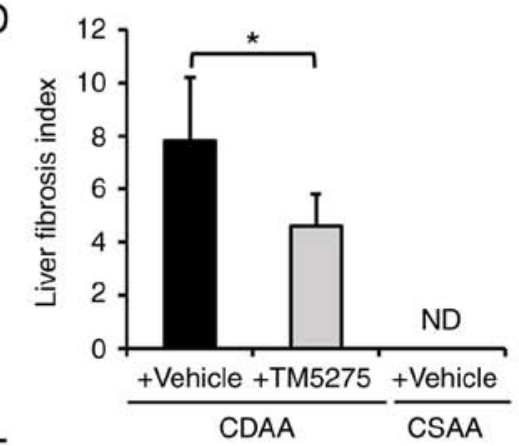

$E$

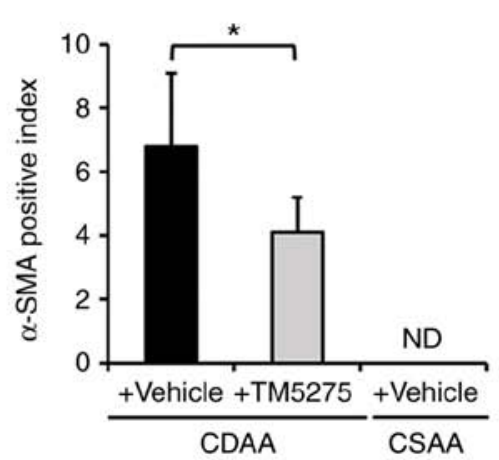

Figure 1. Effects of TM5275 on steatohepatitis in CDAA-fed rats. (A) Schematic of CDAA-induced rat steatohepatitis models. (B) Representative microphotographs of liver sections stained with hematoxylin and eosin, Sirius-Red and $\alpha$-SMA. Scale bar, $50 \mu \mathrm{m}$. (C) Histological scores of steatosis, inflammation and hepatocyte ballooning according to the Nonalcoholic Fatty Liver Disease Activity Score. Semi-quantitation of (D) Sirius-Red and (E) $\alpha$-SMA-positive areas by the National Institutes of Health ImageJ software (version 1.52). All quantitative analyses were performed for 5 fields per each section in high-power fields at $\mathrm{x} 400$ magnification. Data are presented as the mean $\pm \mathrm{SD}(\mathrm{n}=6) .{ }^{*} \mathrm{P}<0.05$ as indicated. CDAA, choline-deficient, L-amino acid-defined diet; SMA, smooth muscle actin; CSAA, choline-supplemented amino acid; ND, not detected.

induced a profound progression of liver fibrosis in diabetic OLETF rats (Fig. 3C). Despite the above insufficiencies in terms of liver enzyme levels and glucose tolerance, it was noteworthy that TM5275 could remarkably suppress PS-mediated liver fibrosis development in OLETF rats in a dose-dependent manner (Fig. 3C and D). As in the CDAA-fed rats, $\alpha$-SMA-immunopositive areas were decreased along with attenuation of liver fibrosis in PS-mediated rats who were administered TM5275 (Fig. 3C and D). These data support the hypothesis that a PAI-1 inhibitor attenuates liver fibrosis through direct action upon activated HSCs.

TM5275 suppresses proliferative and fibrogenic activities in rat activated hepatic stellate cells. Next, we examined the direct effect of TM5275 on HSC-T6 cells, a rat HSC line, in vitro to elucidate the molecular mechanism underlying the antifibrogenic activity of this compound. A cell proliferation assay revealed that recombinant PAI-1 stimulated the proliferation of HSC-T6 cells in a dose-dependent manner, which was efficiently suppressed by treatment with TM5275 (Fig. 4A). Interestingly, TM5275 also suppressed recombinant TGF- $\beta 1$ (rTGF- $\beta 1$ )-mediated HSC-T6 proliferation in a dose-dependent manner (Fig. 4B). Therefore, we next assessed the interaction between TGF- $\beta 1$ and PAI-1 in HSC-T6 cells and found that an rTGF- $\beta 1$-mediated stimulus upregulated mRNA expression of Serpinel, the gene that encodes PAI-1 (Fig. 4C). Moreover, TM5275 significantly inhibited rTGF- $\beta 1$-stimulated upregulation of Serpinel mRNA expression (Fig. 4C). These results indicate that TM5275 has potential to suppress HSC-T6 cell proliferation by inhibiting the upregulation of PAI-1 stimulated by TGF- $\beta 1$. In addition to its effect on cell proliferation, TM5275 markedly repressed the rTGF- $\beta 1$-induced increases in the mRNA levels of the fibrosis-related genes $T g f b l$ and Collal (Fig. 4D and E). Analysis of intracellular signaling revealed that rTGF- $\beta 1$ increased the protein expression of pAKT, pERK1/2, and pSMAD2/3 in HSC-T6 cells, whereas treatment with TM5275 could suppress rTGF- $\beta$-stimulated phosphorylation of AKT but not that of ERK1/2 and SMAD2/3 (Fig. 4F).

\section{Discussion}

Currently, a variety of medical approaches targeting MetS-related liver fibrosis are in the pipeline. Increasing 
A

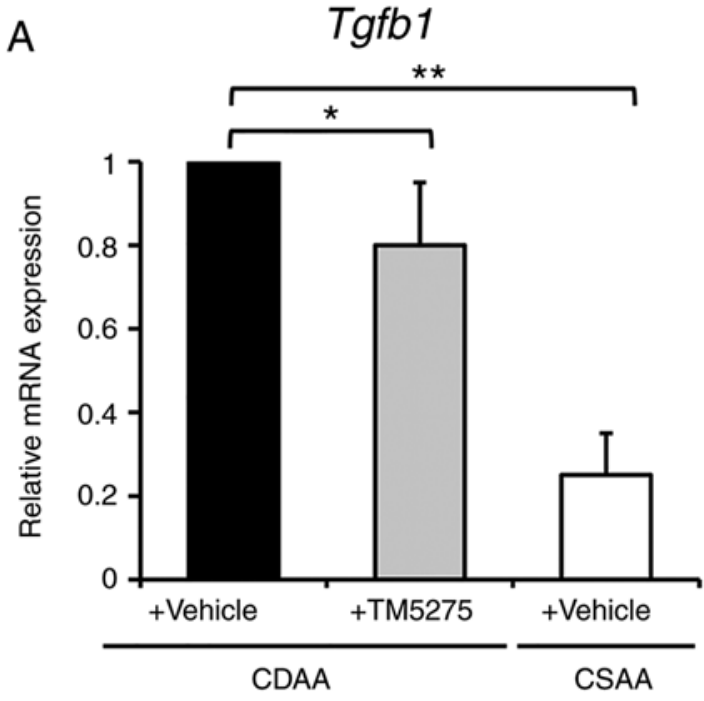

C

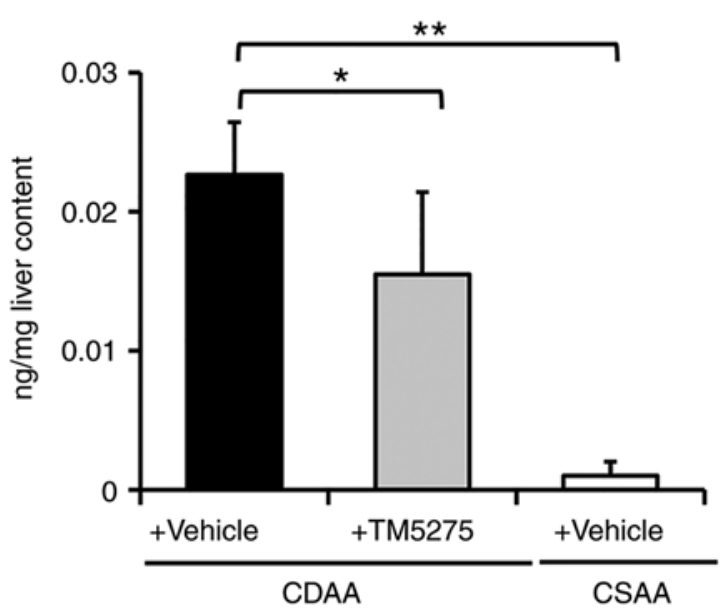

B

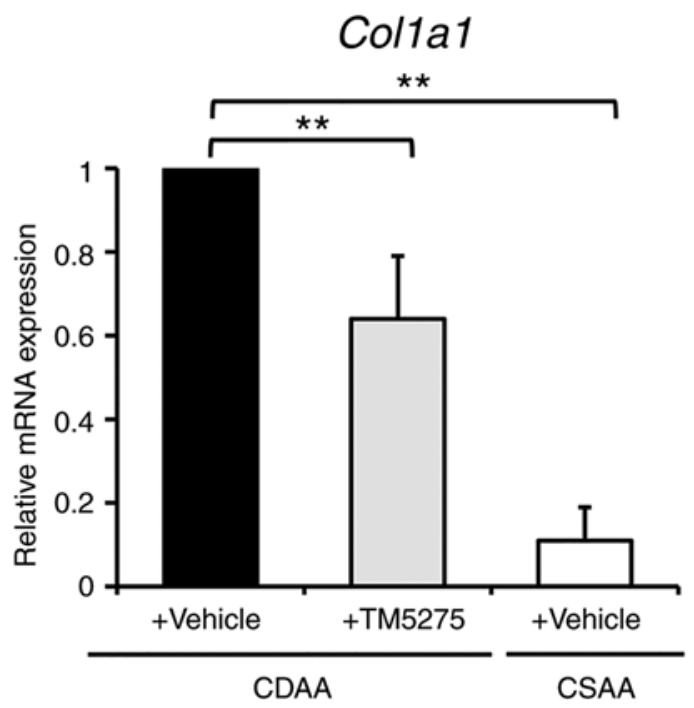

D

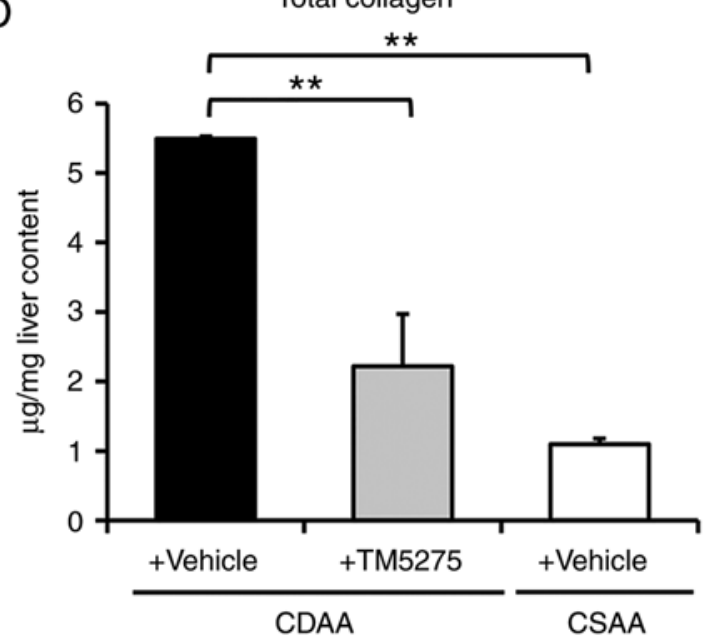

Figure 2. Alterations in fibrotic markers in the livers of CDAA-fed rats. Relative hepatic mRNA levels of (A) Tgfb1 and (B) Collal. (C) Hepatic levels of TGF- $\beta 1$ protein in experimental rats. (D) Hepatic levels of total collagen in experimental rats. Gapdh was used as internal control for reverse transcription-quantitative PCR. Quantitative values are relatively indicated as ratios to the values of CDAA+Vehicle groups $(A$ and $B)$ Data are presented as the mean \pm SD ( $=6$ ). ${ }^{*} \mathrm{P}<0.05$ and ${ }^{* *} \mathrm{P}<0.01$ as indicated. CDAA, choline-deficient, L-amino acid-defined diet; Tgfb1, tumor growth factor $\beta 1 ;$ Colla1, collagen type $1 \alpha 1 ;$ CSAA, choline-supplemented amino acid.

evidence for the ability to address the reversibility of liver fibrosis has led to intense interest in comprehending the regulation of collagen degradation and resolution. It has been recognized that liver fibrosis is characterized by the transdifferentiation of HSCs into activated myofibroblasts, proliferative arrest of hepatocytes, and the accumulation of extracellular matrix (ECM) (25). Thus, a reversion of these sequences has been emphasized as major treatment strategies for liver fibrosis. In the present study, we demonstrated that TM5275, a novel PAI-1 inhibitor, could attenuate liver fibrosis in two mechanistically different models of liver fibrosis, CDAA-fed NASH rats and PS-mediated diabetic rats. The CDAA-fed rats characteristically display a steatohepatitis-based liver fibrosis while they don't show a typical diabetic status including insulin resistance and hyperglycemia (26). On the other hand, PS-mediated diabetic rats develop liver fibrosis with insulin resistance and diabetes, but they show a limited steatosis and inflammation (24). In our results, TM5275 attenuated liver fibrosis development with decreased expressions of $\alpha$-SMA in both models without affecting steatosis and inflammation in CDAA-fed rats as well as insulin resistance in PS-mediated diabetic rats. These suggest the possibility that TM5275 directly affect liver fibrosis development via HSC activation under the MetS condition.

Several studies have suggested that PAI-1 could potentially affect both collagen production and degradation (27-29). It has also been reported that HSCs are a key source of PAI-1 and that there is an imbalance between PAs and PAI-1 at different stages of liver fibrosis development; at an early stage, uPA and expression of its receptor are predominantly elevated, while during the cirrhotic stage, increased expression of PAI-1 hampers uPA activity, which results in the inhibition of plasmin generation and attenuation of excess ECM degradation (30-32). Moreover, PAI-1 has also been shown to regulate the proliferation, adhesion, and migration of various types of cells, which stimulates the migration of leukocytes and collagen-producing cells into damaged tissue (27-29). This indicates that PAI-1 itself might also promote ECM synthesis. 
A
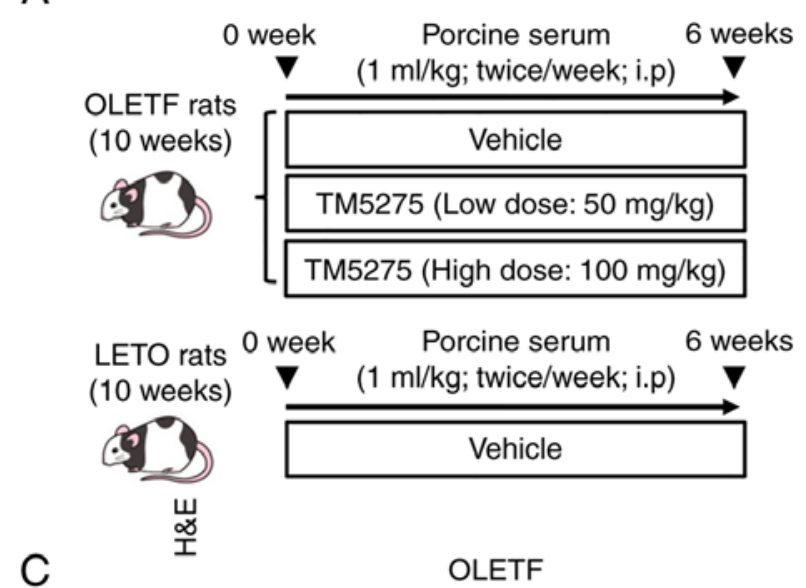

B

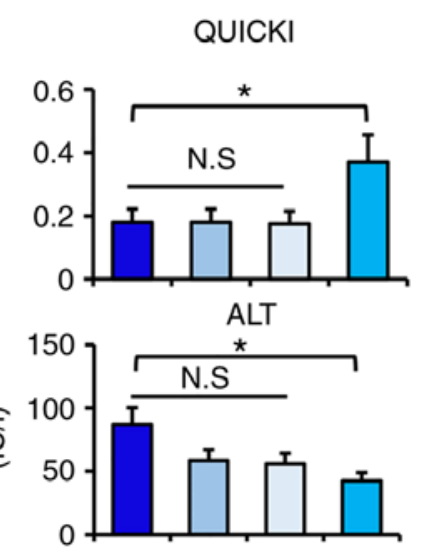

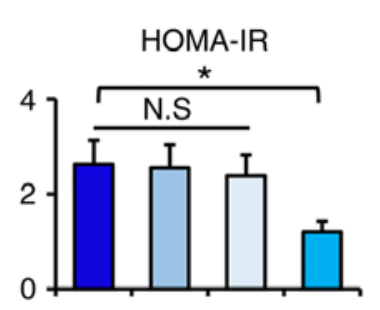

OLETF+Vehicle OLETF+TM5275 (Ld) $\square$ OLETF+TM5275 (Hd) $\square$ LETO+Vehicle
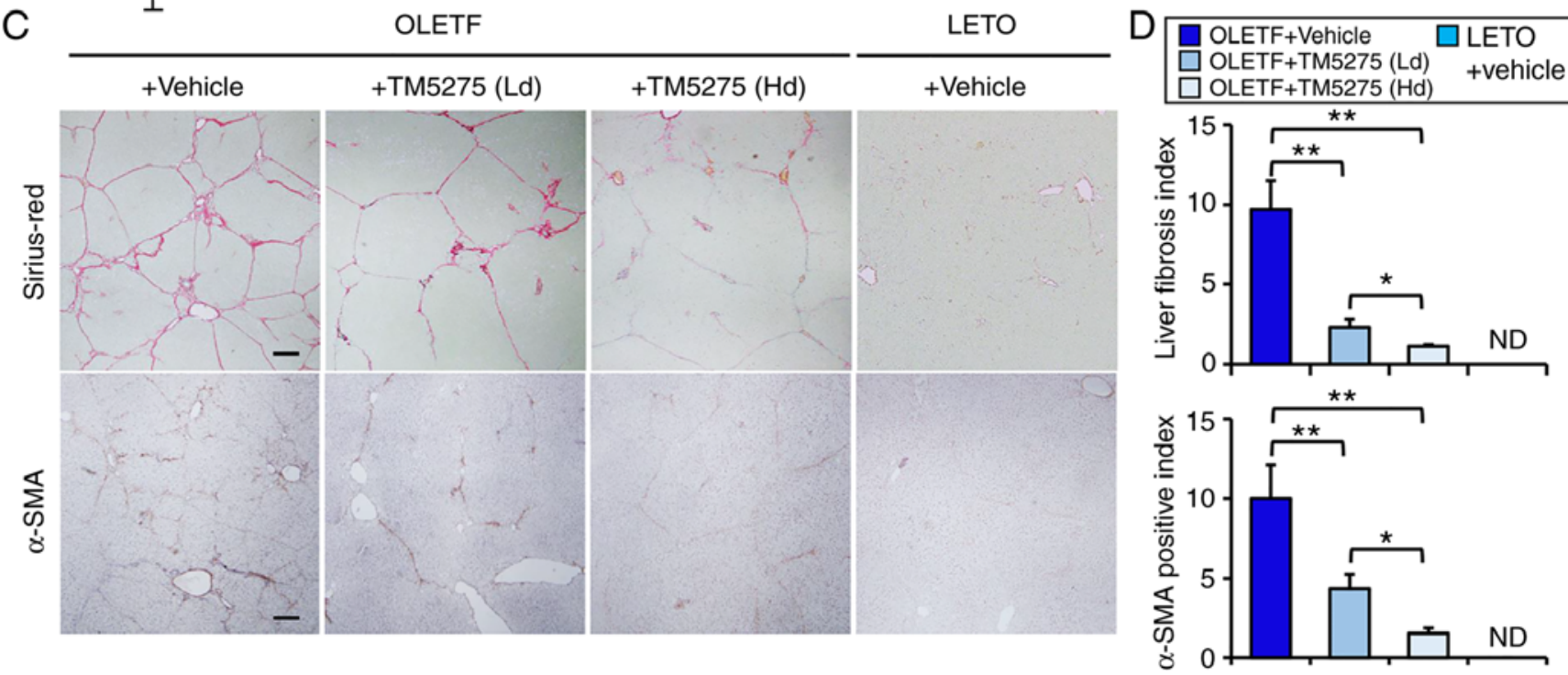

Figure 3. Effects of TM5275 on porcine serum-induced liver fibrosis in diabetic OLETF rats. (A) Schematic of porcine serum-induced liver fibrosis in diabetic rat models. (B) The values of QUICKI, HOMA-IR and serum levels of ALT in experimental rats. (C) Representative images of liver sections stained with Sirius-Red and $\alpha$-SMA. Scale bar, $50 \mu \mathrm{m}$. (D) Semi-quantitation of Sirius Red- and $\alpha$-SMA-positive area by National Institutes of Health ImageJ software (version 1.52). All quantitative analyses were performed for 5 fields per each section in high-power fields at $\mathrm{x} 400 \mathrm{magnification}$. Data are presented as the mean $\pm \mathrm{SD}(\mathrm{n}=6) .{ }^{*} \mathrm{P}<0.05$ and $^{* *} \mathrm{P}<0.01$ as indicated. QUICKI, Quantitative Insulin Sensitivity Check Index; HOMA-IR, homeostasis model assessment-insulin resistance; ALT, alanine aminotransferase; SMA, smooth muscle actin; Ld, low dose; Hd, high dose; N.S., not significant; ND, not detected; OLETF, Otsuka Long-Evans Tokushima Fatty; LETO, Long-Evans Tokushima Otsuka; wk, week.

Actually, our data from in vitro experiments demonstrated that stimulation of recombinant PAI-1 induced the proliferation of HSC-T6 cells in a dose-dependent manner, which was abrogated by treatment with TM5275. These results support the idea that PAI-1 directly augmented the proliferative activity of HSC.

Furthermore, PAI-1 gene expression is regulated by various biological factors including TGF- $\beta$, IL-1 $\beta$, epidermal growth factor, insulin-like growth factor 1, platelet-derived growth factor, and basic fibroblast growth factor (33-37). Of the numerous key regulatory factors, TGF- $\beta 1$ is a principle mediator of HSC activation (25). Previous basic studies of the genetic overexpression of TGF- $\beta$ have revealed a vital contribution of TGF- $\beta 1$ to HSC activation and fibrogenesis $(38,39)$. The TGF- $\beta 1$ signaling pathway is mediated by the SMAD family, but other effectors such as phosphatidylinositol-3 kinase (PI3K), mitogen-activated protein kinase (MAPK), and nuclear factor $\kappa \mathrm{B}(\mathrm{NF}-\kappa \mathrm{B})$, which also have key roles in cell proliferation, apoptosis, differentiation and ECM synthesis may be involved, and can independently regulate SMAD expression (40). Importantly, upon its activation and trans-differentiation, TGF- $\beta 1$ transcriptionally controls the gene expression of PAI- 1 as well as that of $\alpha 1$ and $\alpha 2$ type 1 procollagen, and tissue inhibitor of metalloproteinase-1 and -2 (25). It has been confirmed that TGF- $\beta 1$ induces PAI-1 synthesis, and a TGF- $\beta 1$ response element has been found in the promoter of the PAI-1 gene; in addition, multiple lines of evidence have determined that PAI-1 expression might be regulated in connection with several signaling pathways. For instance, continuous stimulation of TGF- $\beta$ prominently induced not only phosphorylation of Smad2/3 but also upregulation of PAI-1 $(41,42)$. Moreover, TGF- $\beta$ also stimulates the activation of c-Src kinase, which triggers to phosphorylation of caveolin-1, an upstream repressor of EGFR and the RhoA/ROCK pathway and leads to upregulate PAI-1 expression (43). Additionally, in another study, TGF- $\beta$ administration induced progressive fibrosing steatohepatitis in mice, and interestingly, this process was reversed by a 
A

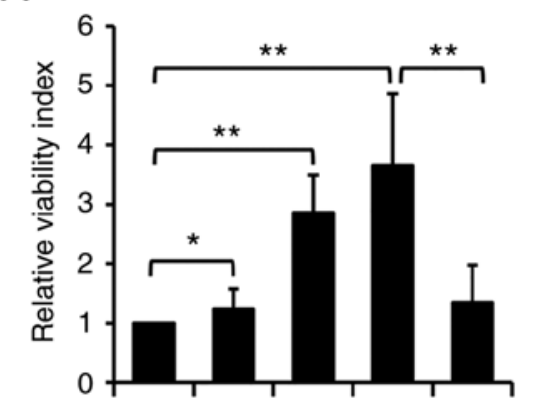

B

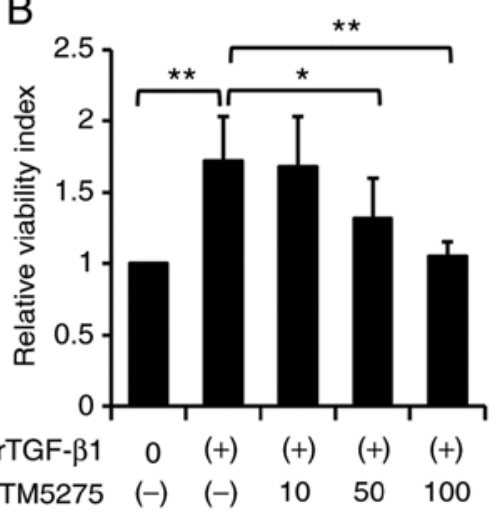

F
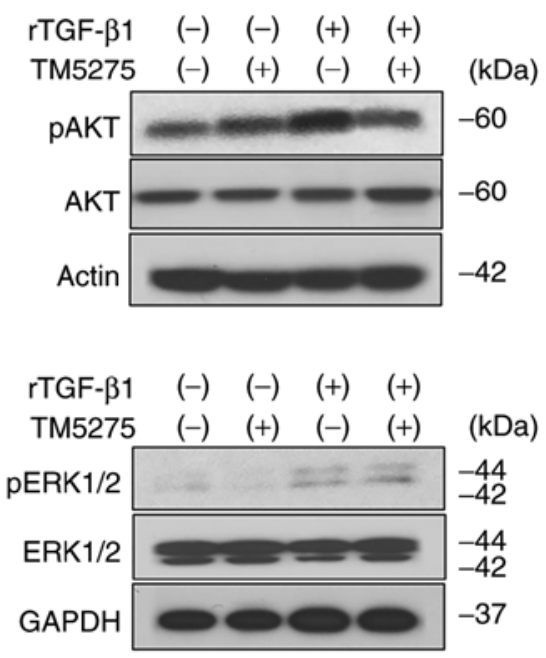

$\begin{array}{lllll}\text { rTGF- } \beta 1 & (-) & (-) & (+) & (+)\end{array}$

$\begin{array}{llllll}\text { TM5275 } & (-) & (+) & (-) & (+) & (\mathrm{kDa})\end{array}$

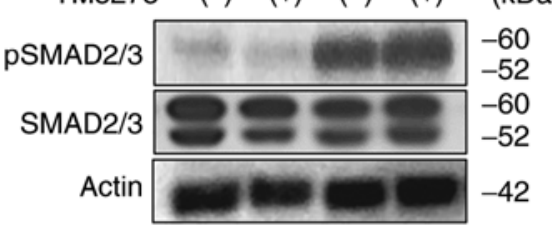

C
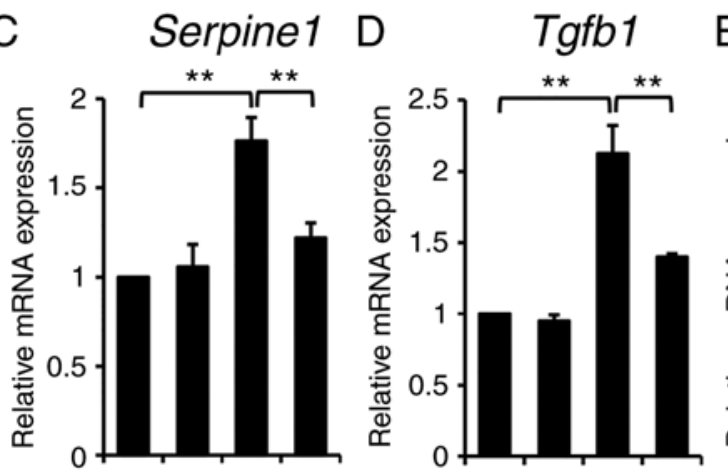

E Col1a1

$\begin{array}{rrrr}\text { rTGF- } \beta 1(-) & (-) & (+) \quad(+)\end{array}$

rTGF- $\beta 1(-) \quad(-)$

$(-)(+)(+)$

(+) $\mathrm{rTGF}-\beta 1(-)$

F- $\beta 1(-)$

(-) $(+)(+)$

TM5275 (-)

$(+)(-)(+)$

TM5275(-) (+)

$(+)(-)(+)$

+) TM5275(-)

$(+) \quad(-) \quad(+)$

Figure 4. Effects of TM5275 on hepatic stellate cell activation. (A) Cell proliferation of HSC-T6 cells treated with and/or without recombinant PAI-1 (0-5 $\mu \mathrm{g} / \mathrm{ml})$ and TM5275 $(100 \mu \mathrm{M})$. (B) Proliferation of HSC-T6 cells treated with and without rTGF- $\beta 1(10 \mathrm{ng} / \mathrm{ml})$ and TM5275 $(0-100 \mu \mathrm{M})$. Relative mRNA levels of (C) Serpine1, (D) Tgfbl and (E) Collal in HSC-T6 cells treated with and/or without rTGF- $\beta 1$ (10 ng/ml) and TM5275 (100 $\mu$ M). Gapdh was used as internal control for reverse transcription-quantitative PCR. (F) Western blots of whole cell lysates from HSC-T6 cells for total and phosphorylated AKT, ERK1/2 and SMAD2/3. HSC-T6 cells were cultured with and/or without rTGF- $\beta 1(10 \mathrm{ng} / \mathrm{ml})$ and TM5275 $(100 \mu \mathrm{M})$. Actin and GAPDH were used as internal control for western blotting. Quantitative values are relatively indicated as ratios to the values of the rPAI-1(-)/TM5275(-) group (A) or the rTGF- $\beta 1(-) /$ TM5275(-) group (B-E) Data are presented as the mean \pm SEM $(n=8)$. ${ }^{*} \mathrm{P}<0.05$ and ${ }^{* *} \mathrm{P}<0.01$ as indicated. rPAI-1, recombinant plasminogen activator inhibitor-1; rTGF- $\beta 1$, recombinant tumor growth factor- $\beta 1$; Collal, collagen type $1 \alpha 1 ; \mathrm{p}$, phosphorylated.

PPAR $\alpha$ agonist, which functioned via AMPK-mediated induction of SHP gene expression with a marked decrease in the mRNA and protein expression of PAI-1 and other fibrotic markers (44). In line with these findings, the results of our in vitro experiments showed that stimulation with recombinant TGF- $\beta 1$ significantly increased Serpinel gene expression in HSC-T6 cells, which was abrogated by treatment with TM5275. Consistently, with decreased Serpinel expression, TM5275 significantly suppressed TGF- $\beta 1$-stimulated HSCs proliferation. Interestingly, we discovered that TM5275 inhibited the phosphorylation of AKT stimulated by TGF- $\beta 1$, but it did not alter the phosphorylation of ERK1/2 and SMAD2/3. We speculate the possibility that TM5275 may directly PAI-1/PI3K/AKT pathway in HSC-T6 cells because of its suppressive effect PAI-1-stimulated HSC-T6 cell viability. The definitive molecular mechanism responsible for these heterogenous effects of TM5275 on TGF- $\beta 1$ signaling in HSCs has been uncertain until now, as PAI-1 seemed to be multifunctional and have multiple interactions.

Several limitations are apparent in the present study. First, the functional role of PAI-1 in liver fibrosis development is still controversial, although PAI-1 has been found to be involved in the process of fibrotic progression in various tissues. For example, a recent report showed that either genetic deletion or pharmacologic inhibition of PAI-1 ameliorated methionineand choline-deficient diet-induced hepatic steatosis but did not affect hepatic inflammation or fibrosis (21). These conflicting effects of PAI-1 inhibition on steatohepatitis suggest that the impact of PAI-1 regulation on tissue fibrosis, especially in the liver, is highly dependent on the tissue type and experimental model. Second, this study elucidated the preventive effects of a PAI-1 inhibitor on the development of liver fibrosis, while the pharmacological properties of fibrinolysis and liver regeneration in an established model of liver fibrosis are still obscure. Future studies should address whether TM5275 could induce fibrinolysis and efficient liver regeneration in other models of cirrhosis.

Taken together, our data show that TM5275, a novel PAI-1 inhibitor, appears to have a protective effect on liver fibrosis development in rat fibrosis models under the condition of MetS. Notably, this agent has the potential to directly suppress HSC proliferation and profibrogenic activity by blockade of TGF- $\beta /$ PAI-1 binding. These findings suggest a possibility that TM5275 confers a clinical benefit for liver fibrosis with MetS, but there is a major issue to be addressed for future clinical application. Representatively, this agent has a risk to foster 
the bleeding tendency especially in cirrhotic patients because of its antithrombotic action. Further studies are warranted to determine the influences of PAI-1 inhibitor on other organs in chronic liver diseases.

\section{Acknowledgements}

Not applicable.

\section{Funding}

The present study was supported by Japan Society for the Promotion of Science KAKENHI (grant no. JP2646101).

\section{Availability of data and materials}

The datasets used and/or analyzed during the current study are available from the corresponding author on reasonable request.

\section{Author's contributions}

RN, KK and HY conceived and designed the current study, and outlined the proof. RN, KK and MK designed the model and the computational framework, and analyzed the data. RN, TN, KM, HK, MK, HT, YA, AD, KA and NN contributed to animal breeding, sample collection and performed the experiments. $\mathrm{RN}$ and $\mathrm{KK}$ validated the results. TM provided the TM5275 compound and interpreted the data. RN and KK wrote the manuscript in consultation with TM and HY. HY supervised the current study. RN acquired the funding. All authors read and approved the final manuscript.

\section{Ethics approval and consent to participate}

All animal procedures were performed according to the criteria outlined in the Guide for the Care and Use of Laboratory Animals prepared by the National Academy of Sciences. All experiments were approved by the Animal Care and Use Committee of Nara Medical University (protocol no. 10034).

\section{Patient consent for publication}

Not applicable.

\section{Competing interests}

The authors declare that they have no competing interests.

\section{References}

1. Farrell GC and Larter CZ: Nonalcoholic fatty liver disease: From steatosis to cirrhosis. Hepatology 43: S99-S112, 2006.

2. Angulo P: Nonalcoholic fatty liver disease. N Engl J Med 346 : 1221-1231, 2002.

3. Sanyal AJ, Neuschwander-Tetri BA and Tonascia L: End points must be clinically meaningful for drug development in nonalcoholic fatty liver disease. Gastroenterology 150: 11-13, 2016.

4. Younossi ZM, Koenig AB, Abdelatif D, Fazel Y, Henry L and Wymer M: Global epidemiology of nonalcoholic fatty liver disease-Meta-analytic assessment of prevalence, incidence, and outcomes. Hepatology 64, 73-84, 2016.
5. Dulai PS, Singh S, Patel J, Soni M, Prokop LJ, Younossi Z, Sebastiani G, Ekstedt M, Hagstrom H, Nasr P, et al: Increased risk of mortality by fibrosis stage in nonalcoholic fatty liver disease: Systematic review and meta-analysis. Hepatology 65: 1557-1565, 2017.

6. Angulo P, Kleiner DE, Dam-Larsen S, Adams LA, Bjornsson ES, Charatcharoenwitthaya P, Mills PR, Keach JC, Lafferty HD, Stahler A, et al: Liver fibrosis, but No other histologic features, is associated with long-term outcomes of patients with nonalcoholic fatty liver disease. Gastroenterology 149: 389-397 e10, 2015.

7. Erickson LA, Ginsberg MH and Loskutoff DJ: Detection and partial characterization of an inhibitor of plasminogen activator in human platelets. J Clin Invest 74: 1465-1472, 1984.

8. Providence KM and Higgins PJ: PAI-1 expression is required for epithelial cell migration in two distinct phases of in vitro wound repair. J Cell Physiol 200: 297-308, 2004.

9. Toriseva $\mathrm{M}$ and Kähäri VM: Proteinases in cutaneous wound healing. Cell Mol Life Sci 66: 203-224, 2009.

10. Lazar MH, Christensen PJ, Du M, Yu B, Subbotina NM, Hanson KE, Hansen JM, White ES, Simon RH and Sisson TH: Plasminogen activator inhibitor-1 impairs alveolar epithelial repair by binding to vitronectin. Am J Respir Cell Mol Biol 31: 672-678, 2004

11. Takeshita K, Hayashi M, Iino S, Kondo T, Inden Y, Iwase M, Kojima T, Hirai M, Ito M, Loskutoff DJ, et al: Increased expression of plasminogen activator inhibitor-1 in cardiomyocytes contributes to cardiac fibrosis after myocardial infarction. Am J Pathol 164: 449-456, 2004.

12. Ma LJ and Fogo AB: PAI-1 and kidney fibrosis. Front Biosci (Landmark Ed) 14: 2028-2041, 2009.

13. Clouthier DE, Comerford SA and Hammer RE: Hepatic fibrosis, glomerulosclerosis, and a lipodystrophy-like syndrome in PEPCK-TGF-betal transgenic mice. J Clin Invest 100: 2697-2713, 1997.

14. Wang H, Zhang Y and Heuckeroth RO: PAI-1 deficiency reduces liver fibrosis after bile duct ligation in mice through activation of tPA. FEBS Lett 581: 3098-3104, 2007.

15. Ajmera V, Perito ER, Bass NM, Terrault NA, Yates KP, Gill R, Loomba R, Diehl AM and Aouizerat BE; NASH Clinical Research Network: Novel plasma biomarkers associated with liver disease severity in adults with nonalcoholic fatty liver disease. Hepatology 65: 65-77, 2017.

16. Hu PF, Chen H, Zhong W, Lin Y, Zhang X, Chen YX and Xie WF: Adenovirus-mediated transfer of siRNA against PAI-1 mRNA ameliorates hepatic fibrosis in rats. J Hepatol 51: 102-113, 2009.

17. Izuhara $\mathrm{Y}$, Yamaoka N, Kodama H, Dan T, Takizawa S, Hirayama N, Meguro K, van Ypersele de Strihou C and Miyata T: A novel inhibitor of plasminogen activator inhibitor-1 provides antithrombotic benefits devoid of bleeding effect in nonhuman primates. J Cereb Blood Flow Metab 30: 904-912, 2010.

18. Tashiro Y, Nishida C, Sato-Kusubata K, Ohki-Koizumi M, Ishihara M, Sato A, Gritli I, Komiyama H, Sato Y, Dan T, et al: Inhibition of PAI-1 induces neutrophil-driven neoangiogenesis and promotes tissue regeneration via production of angiocrine factors in mice. Blood 119: 6382-6393, 2012.

19. Imai J, Yahata T, Ichikawa H,Ibrahim AA, Yazawa M,Sumiyoshi $H$, Inagaki Y, Matsushima M, Suzuki T, Mine T, Ando K, et al: Inhibition of plasminogen activator inhibitor-1 attenuates against intestinal fibrosis in mice. Intest Res 18: 219-228, 2020.

20. Lee SM, Dorotea D, Jung I, Nakabayashi T, Miyata T and $\mathrm{Ha} \mathrm{H}$ : TM5441, a plasminogen activator inhibitor-1 inhibitor, protects against high fat diet-induced non-alcoholic fatty liver disease. Oncotarget 8: 89746-89760, 2017.

21. Henkel AS, Khan SS, Olivares S, Miyata T and Vaughan DE: Inhibition of plasminogen activator inhibitor 1 attenuates hepatic steatosis but does not prevent progressive nonalcoholic steatohepatitis in mice. Hepatol Commun 2: 1479-1492, 2018.

22. Katz A, Nambi SS, Mather K, Baron AD, Follmann DA, Sullivan G and Quon MJ: Quantitative insulin sensitivity check index: A simple, accurate method for assessing insulin sensitivity in humans. J Clin Endocrinol Metab 85: 2402-2410, 2000.

23. Kleiner DE, Brunt EM, Van Natta M, Behling C, Contos MJ, Cummings OW, Ferrell LD, Liu YC, Torbenson MS, Unalp-Arida A, et al: Design and validation of a histological scoring system for nonalcoholic fatty liver disease. Hepatology 41 : 1313-1321, 2005

24. Kaji K, Yoshiji H, Kitade M, Ikenaka Y, Noguchi R, Yoshii J, Yanase K, Namisaki T, Yamazaki M, Moriya K, et al: Impact of insulin resistance on the progression of chronic liver diseases. Int J Mol Med 22: 801-808, 2008. 
25. Tsuchida $T$ and Friedman SL: Mechanisms of hepatic stellate cell activation. Nat Rev Gastroenterol Hepatol 14: 397-411, 2017.

26. Ozutsumi T, Namisaki T, Shimozato N, Kaji K, Tsuji Y, Kaya D, Fujinaga Y, Furukawa M, Nakanishi K, Sato S, et al: Combined treatment with sodium-glucose cotransporter-2 inhibitor (Canagliflozin) and dipeptidyl peptidase-4 inhibitor (teneligliptin) alleviates NASH progression in A non-diabetic rat model of steatohepatitis. Int J Mol Sci 21: 2164, 2020.

27. Gleizes PE, Munger JS, Nunes I, Harpel JG, Mazzieri R, Noguera I and Rifkin DB: TGF-beta latency: Biological significance and mechanisms of activation. Stem Cells 15: 190-197, 1997.

28. Mulligan-Kehoe MJ, Schwartz GN and Zacharski LR: The functions of plasminogen activator inhibitor-1: Do we have all the pieces of PAI? Thromb Res 117: 483-486, 2006.

29. Bajou K, Noël A, Gerard RD, Masson V, Brunner N, Holst-Hansen C, Skobe M, Fusenig NE, Carmeliet P, Collen D and Foidart JM: Absence of host plasminogen activator inhibitor 1 prevents cancer invasion and vascularization. Nat Med 4: 923-928, 1998.

30. Zhang LP, Takahara T, Yata Y, Furui K, Jin B, Kawada N and Watanabe A: Increased expression of plasminogen activator and plasminogen activator inhibitor during liver fibrogenesis of rats: Role of stellate cells. J Hepatol 31: 703-711, 1999.

31. Seki T, Imai H, Uno S, Ariga T and Gelehrter TD: Production of tissue-type plasminogen activator (t-PA) and type-1 plasminogen activator inhibitor (PAI-1) in mildly cirrhotic rat liver. Thromb Haemost 75: 801-807, 1996.

32. Leyland H, Gentry J, Arthur MJ and Benyon RC: The plasminogen-activating system in hepatic stellate cells. Hepatology 24 $1172-1178,1996$.

33. Dennler S, Itoh S, Vivien D, ten Dijke P, Huet S and Gauthier JM: Direct binding of Smad3 and Smad4 to critical TGF beta-inducible elements in the promoter of human plasminogen activator inhibitor-type 1 gene. EMBO J 17: 3091-3100, 1998.

34. Okada H, Woodcock-Mitchell J, Mitchell J, Sakamoto T, Marutsuka K, Sobel BE and Fujii S: Induction of plasminogen activator inhibitor type 1 and type 1 collagen expression in rat cardiac microvascular endothelial cells by interleukin-1 and its dependence on oxygen-centered free radicals. Circulation 97: 2175-2182, 1998.

35. Crandall DL, Groeling TM, Busler DE and Antrilli TM: Release of PAI-1 by human preadipocytes and adipocytes independent of insulin and IGF-1. Biochem Biophys Res Commun 279: 984-988, 2000.

36. Wilkins-Port CE, Ye Q, Mazurkiewicz JE and Higgins PJ: TGF-beta1 + EGF-initiated invasive potential in transformed human keratinocytes is coupled to a plasmin/MMP-10/ MMP-1-dependent collagen remodeling axis: Role for PAI-1. Cancer Res 69: 4081-4091, 2009.
37. Su EJ, Fredriksson L, Schielke GP, Eriksson U and Lawrence DA Tissue plasminogen activator-mediated PDGF signaling and neurovascular coupling in stroke. J Thromb Haemost 7 (Suppl 1): S155-S158, 2009.

38. Hellerbrand C, Stefanovic B, Giordano F, Burchardt ER and Brenner DA: The role of TGFbeta1 in initiating hepatic stellate cell activation in vivo. J Hepatol 30: 77-87, 1999.

39. Kanzler S, Lohse AW, Keil A, Henninger J, Dienes HP, Schirmacher P, Rose-John S, zum Büschenfelde KH and Blessing M: TGF-betal in liver fibrosis: An inducible transgenic mouse model to study liver fibrogenesis. Am J Physiol 276: G1059-G1068, 1999.

40. Derynck R and Zhang YE: Smad-dependent and Smad-independent pathways in TGF-beta family signalling. Nature 425: 577-584, 2003

41. Motojima M,Kakuchi J and Yoshioka T: Association of TGF-beta signaling in angiotensin II-induced PAI-1 mRNA upregulation in mesangial cells: Role of PKC. Biochim Biophys Acta 1449: 217-226, 1999.

42. Dong C, Zhu S, Alvarez RJ and Goldschmidt-Clermont PJ: Angiotensin II induces PAI-1 expression through MAP kinase-dependent, but TGF beta and PI3 kinase-independent pathway. J Heart Lung Transplant 20: 226-227, 2001.

43. Samarakoon R, Higgins SP, Higgins CE and Higgins PJ: TGF-beta1-induced plasminogen activator inhibitor-1 expression invascularsmoothmusclecellsrequirespp60(c-src)/EGFR(Y845) and Rho/ROCK signaling. J Mol Cell Cardiol 44: 527-538, 2008.

44. Chanda D, Lee CH, Kim YH, Noh JR, Kim DK, Park JH, Hwang JH, Lee MR, Jeong KH, Lee IK, et al: Fenofibrate differentially regulates plasminogen activator inhibitor-1 gene expression via adenosine monophosphate-activated protein kinase-dependent induction of orphan nuclear receptor small heterodimer partner. Hepatology 50: 880-892, 2009 .

This work is licensed under a Creative Commons Attribution-NonCommercial-NoDerivatives 4.0 International (CC BY-NC-ND 4.0) License. 